\title{
Droop Control for Islanded Microgrids with Compensating Approach
}

\author{
M. I. Azim and M. A. Hossain \\ School of Engineering and \\ Information Technology \\ The University of New South Wales \\ Canberra, ACT 2610, Australia \\ m.azim@student.unsw.edu.au, \\ md.hossain6@student.adfa.edu.au
}

\author{
M. J. Hossain \\ Griffith School of Engineering \\ Griffith University, Gold Coast \\ QLD 4222, Australia \\ j.hossain@griffith.edu.au
}

\author{
H. R. Pota \\ School of Engineering and \\ Information Technology \\ The University of New South Wales \\ Canberra, ACT 2610, Australia \\ h.pota@adfa.edu.au
}

\begin{abstract}
This paper proposes a droop control algorithm to distribute load changes automatically between two invertersourced generators in an islanded microgrid. A compensating approach is added to the conventional droop control scheme in order to add a power offset to the active power so that the output powers of the inverters remain balanced if the output of renewable energy varies. The droop control parameters are chosen based on eigenvalue analysis obtained from linear modal analysis. In this paper, it is shown that the proposed control method ensures effective power sharing and provides good performance under diverse operating conditions without the need for communication.
\end{abstract}

Index Terms-renewable energy source, voltage-source converter (VSC), microgrid, droop control, compensating approach, real power sharing, reactive power sharing.

\section{INTRODUCTION}

This paper focuses on an accurate decentralized droop control algorithm that can compensate the effect of the variation of renewable energy sources (RES)-based inverter input and share both active and reactive powers effectively when the renewable energy output varies and the load changes.

Presently fossil fuels are the main source of energy in different countries including Australia. Some environmental and economical concerns are caused by their use [1]. Renewable energy sources provide a better solution that can be installed as distributed-generation (DG) units [2] and offer many advantages such as environmental friendly operation, reduction of transmission and distribution losses and costs, higher energy efficiency and other economic benefits [3]. The operation and planning of the electrical network can be affected by the growing share of DG units [4]. This suggests a coordinated approach to integrate DG units, resulting in the concept of microgrid [5].

A microgrid consists of distributed energy system, such as loads and resources [6], [7]. It is also a controllable system, which is the key advantage in case of power networks [8]. In most cases, resources are connected to microgrid via voltage source converters, such as inverters, and microgrid is connected to the load-side at the point of common coupling (PCC) [9]. Microgrids do not have the rotating inertia [9] and aggregated loads like conventional grids [10]. For stable operation of microgrids, various control approaches are used with or without communication [11], [12].

In recent years, different controllers for microgrids have been used. One of the schemes is droop-based control; in which controllers operate in an automatic process and provide reliable operation [13]. Conventional droop control consists of active power/frequency (P/f) control and reactive power/voltage $(\mathrm{Q} / \mathrm{V})$ control [14], that means active power sharing is based on frequency droop control [15]. Modern inverters are able to change the phase of the output rapidly and specifying the active power sharing control as a rate-ofchange of angle droop control is more meaningful [16].

Most droop controllers are designed and implemented for VSC-connected microgrid by assuming instantaneous change in the output of the renewable energy source as the output requirements change. In reality, there is a delay before the output can change and during that interval a buffer, such as a capacitor, supplies or absorbs the difference in the inverter output power and the generated power. If it fails to buffer the mismatch adequately, a feedback controller can be utilized. In [17], an interesting control scheme has been proposed, although its implementation in effective load sharing has not been clarified. It also claims that the dc gain of the compensator, used for balancing the output power of the microgrid, should be unity but this does not result in sharing the RES power proportionally. The value of dc gain depends on the microgrid structure.

Since microgrids do not support the conventional method of reactive power sharing according to the generator terminal voltage [18], a linear model in terms of only output voltage of inverters has been used in [16]. It needs extension to include the impact of the phase angle of output voltage of inverters on reactive power sharing. Both the issues are taken into consideration in this paper.

The organization of the paper is as follows: Section II discusses the modeling of VSC-connected microgrid; system structure with the proposed control strategy and linearization are provided in Section III; Section IV contains the design steps for the proposed controller; controller performance is 
evaluated in Section $\mathrm{V}$ and concluding remarks are outlined in Section VI.

\section{MiCROGRID MODELING}

The basics of microgrid operations and research problems can be analyzed with the help of a two-source microgrid. Voltage source with controllable voltage magnitude and phase represents inverter-connected renewable energy generation [19]. The block diagram of two inverter-interfaced voltagesource microgrid with one load has been illustrated in Fig. 1. In this figure, $P_{d c_{1}}$ and $P_{d c_{2}}$ are the power outputs of two renewable energy sources. dc-link capacitors are used as buffer between generators and inverters. The VSC blocks convert dc voltages into ac. The inverters are fast and there are no stator transients. The voltage-sources are represented by $V_{1} \angle \delta_{1}$ and $V_{2} \angle \delta_{1}$ respectively.

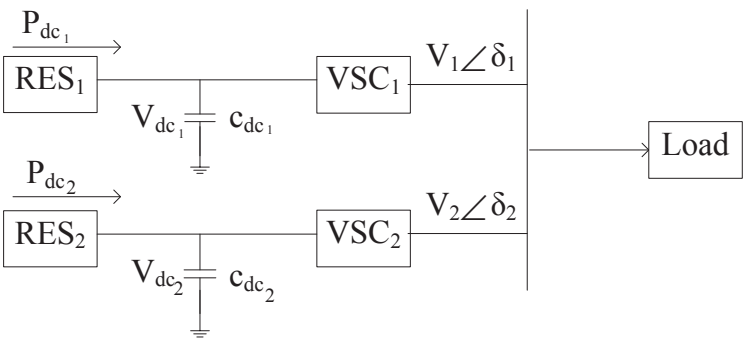

Fig. 1: Block Diagram of a Simple Islanded Microgrid

\section{System Structure With CONTROL}

This section contains the single line diagram, as seen in Fig. 2, of the considered non-linear microgrid system with linearization and control.

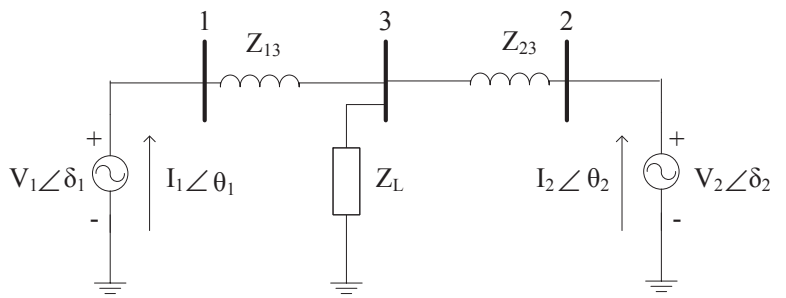

Fig. 2: Single Line Diagram of a Simple Islanded Microgrid

The currents and bus voltages in the microgrid are related as:

$$
\begin{gathered}
Y_{\mathrm{b} u s} V_{\mathrm{b} u s}=I_{\mathrm{b} u s} \\
Y_{\mathrm{b} u s}=\left[\begin{array}{ccc}
\frac{1}{Z_{13}} & 0 & -\frac{1}{Z_{13}} \\
0 & \frac{1}{Z_{23}} & -\frac{1}{Z_{23}} \\
-\frac{1}{Z_{13}} & -\frac{1}{Z_{23}} & \frac{1}{Z_{13}}+\frac{1}{Z_{23}}+\frac{1}{Z_{L}}
\end{array}\right] \\
V_{\mathrm{b} u s}=\left[\begin{array}{l}
V_{1} \angle \delta_{1} \\
V_{2} \angle \delta_{2} \\
V_{3} \angle \delta_{3}
\end{array}\right] ; I_{\mathrm{b} u s}=\left[\begin{array}{l}
I_{1} \angle \theta_{1} \\
I_{2} \angle \theta_{2} \\
I_{3} \angle \theta_{3}
\end{array}\right]
\end{gathered}
$$

\section{A. System Parameters}

The transmission line parameters are $Z_{13}=\mathrm{j} 0.25 \mathrm{pu}$ and $Z_{23}=\mathrm{j} 0.05 \mathrm{pu}$.

\section{B. Load-flow Analysis}

Load-flow analysis is used to determine the initial set values of different bus parameters. It is assumed that bus- 1 is a generator bus, bus- 2 is a slack or reference bus and bus- 3 is a load bus. Considered initial set values: $P_{1}^{0}=0.5 \mathrm{pu}, V_{1}^{0}$ $=1 \mathrm{pu}, V_{2}^{0}=1 \mathrm{pu}, \delta_{2}^{0}=0^{0}$ and load resistor, $Z_{L}^{0}=1 \mathrm{pu}$.

Load- flow solution gives the initial set values of $\delta_{1}, V_{3}$, and $\delta_{3}$; these are: $\delta_{1}^{0}=5.7663^{0}, V_{3}^{0}=0.9984 \mathrm{pu}, \delta_{3}^{0}=-1.4258^{0}$. Using (1), $I_{1}^{0}=0.5014 \mathrm{pu}, \theta_{1}^{0}=1.4544^{0}, I_{2}^{0}=0.4983 \mathrm{pu}, \theta_{2}^{0}$ $=-4.3241^{0}, P_{2}^{0}=0.4969 \mathrm{pu}, P_{3}^{0}=P_{Z_{L}}^{0}=0.9969 \mathrm{pu}, Q_{1}^{0}=$ $0.0377 \mathrm{pu}, Q_{2}^{0}=0.0376 \mathrm{pu}$.

\section{Proposed Control Strategy}

In this sub-section, the background of droop control and its modification for the purpose of sharing the load in a desired manner are described. The active and reactive powers are expressed by the following equations [20]:

$$
\begin{aligned}
S_{12} & =P_{12}+j Q_{12} \\
P_{12} & =\frac{V_{1}}{R_{12}^{2}+X_{12}^{2}}\left[R_{12} V_{1}-R_{12} V_{2} \cos \left(\delta_{1}-\delta_{2}\right)\right. \\
& \left.+X_{12} V_{2} \sin \left(\delta_{1}-\delta_{2}\right)\right] \\
Q_{12} & =\frac{V_{1}}{R_{12}^{2}+X_{12}^{2}}\left[X_{12} V_{1}-X_{12} V_{2} \cos \left(\delta_{1}-\delta_{2}\right)\right. \\
& \left.-R_{12} V_{2} \sin \left(\delta_{1}-\delta_{2}\right)\right]
\end{aligned}
$$

For overhead transmission lines, reactance is greater than resistance, i.e., $X_{12}>R_{12}$. Consequently, $R_{12}$ may be neglected. If the difference between power angles, $\delta_{1}-\delta_{2}$ is small, $\sin \left(\delta_{1}-\delta_{2}\right)=\delta_{1}-\delta_{2}=\Delta \delta$ and $\cos \left(\delta_{1}-\delta_{2}\right)=1$. (4) and (5) then become,

$$
\begin{gathered}
\Delta \delta=\frac{X_{12} P_{12}}{V_{1} V_{2}} \\
V_{1}-V_{2}=\Delta V=\frac{X_{12} Q_{12}}{V_{1}}
\end{gathered}
$$

Based on these relations, the droop equations are [21] $(i=1,2)$ :

$$
\begin{aligned}
\Delta \dot{\delta}_{i} & =-k_{p_{i}}\left(P_{m_{i}}-P_{i}^{0}\right) \\
\Delta V_{i} & =-k_{q_{i}}\left(Q_{m_{i}}-Q_{i}^{0}\right)
\end{aligned}
$$

where, subscript $m$ stands for measured values.

This paper modifies the traditional droop control strategy, known as droop control, with compensation. This is because of ascertaining the balance in the output active power of the microgrid by changing it according to the change in the dc bus voltage of the power sources, $V_{d c_{i}}$. For this purpose power offset, which is added to the output active power, is expressed as a function of the variation of dc-link voltage [17]. The proposed droop equations $(i=1,2)$ :

$$
P_{f t_{i}}^{0}=k_{d c_{i}}\left(V_{d c}^{0}-V_{d c_{i}}\right)+k_{i} \int\left(V_{d c}^{0}-V_{d c_{i}}\right) d t
$$

where, $P_{f t_{i}}^{0}$ is the offset power. Proportional control controls $\left(V_{d c}^{0}-V_{d c_{i}}\right)$ to a constant, while integral control keeps it 
equal to a pre-defined value. In this paper, $k_{i}=0$.

$$
\Delta \dot{\delta}_{i}=-k_{p_{i}}\left(P_{m_{i}}+P_{f t_{i}}^{0}-P_{i}^{0}\right) ; \Delta V_{i}=-k_{q_{i}}\left(Q_{m_{i}}-Q_{i}^{0}\right)
$$

where, $w_{\mathrm{f}}=12 \mathrm{rad} \mathrm{s}^{-1}, k_{p_{1}}=1.2, k_{p_{2}}=6, k_{q_{1}}=0.0012$ and $k_{q_{2}}=0.006$. These parameters are chosen in accordance with the eigenvalue analysis of the linearized closed-loop system being presented in the next sub-section.

Let assume real and reactive power measurement sensors have a first-order dynamics and their transfer functions [16] $(i=1,2)$ :

$$
\frac{P_{m_{i}}(s)}{P_{i}(s)}=\frac{w_{\mathrm{f}}}{s+w_{\mathrm{f}}} ; \frac{Q_{m_{i}}(s)}{Q_{i}(s)}=\frac{w_{\mathrm{f}}}{s+w_{\mathrm{f}}}
$$

In Fig. 3, the proposed droop controller controls voltage and phase angle of dc/ac converter-connected voltage-source, which is connected in an islanded microgrid, when either renewable generation, load or both vary. For example, if load changes, the variation between original load and measured load change is multiplied by droop gains and the controller controls it in a way that angle difference, voltages, real powers and reactive powers of the system remain stable. Same process is done for $\mathrm{dc}$ bus voltage variation. The droop control equations are denoted in this diagram by means of summations and gains.

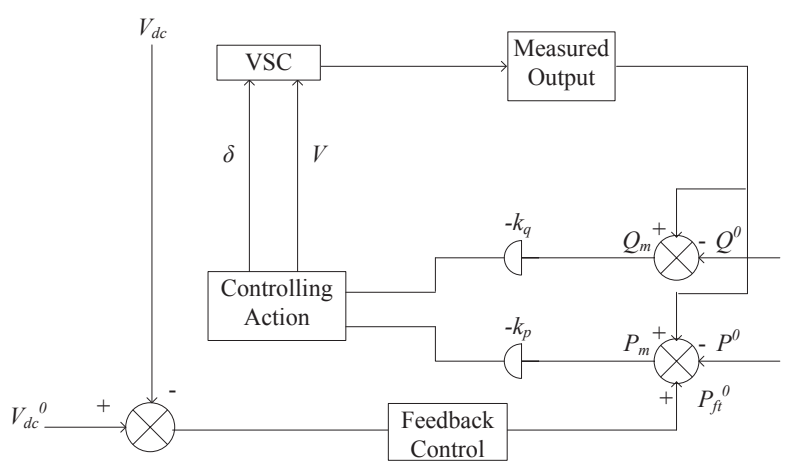

Fig. 3: The Proposed Droop Control Scheme

\section{Linear Modal Analysis}

Linear modal analysis of the non-linear inductive microgrid with the controller is carried out in this paper to tune control parameters and to attain a better understanding of the dynamics of the microgrids. The complex power can be written as $(i, j=$ $1,2 ; j \neq i)$ :

$$
\begin{aligned}
S_{i} & =P_{i}+j Q_{i}=V_{i} \angle \delta_{i} \times I_{i} \angle-\theta_{i} \\
I_{i} \angle-\theta_{i} & =\sum_{j=1}^{2} Y_{\text {reduced }_{i j}} V_{j} \angle \delta_{j} \\
Y_{\text {reduced }_{i j}} & =Y_{i j}-Y_{i 2} Y_{22}^{-1} Y_{2 j}
\end{aligned}
$$

Two voltage-sources and the load bus are grouped in ' 1 ' and '2' respectively to make $Y_{i j}$ from $Y_{\mathrm{b} u s}$ and if it is considered that $Y_{\text {reduced }}{ }_{i j}=G_{i j}+j B_{i j}, \delta_{i j}=\delta_{i}-\delta_{j}$, then putting (14) and (15) together [16]:

$$
\begin{aligned}
P_{i} & =\sum_{j=1}^{2} V_{i} V_{j}\left(G_{i j} \cos \delta_{i j}+B_{i j} \sin \delta_{i j}\right) \\
Q_{i} & =\sum_{j=1}^{2} V_{i} V_{j}\left(G_{i j} \sin \delta_{i j}-B_{i j} \cos \delta_{i j}\right)
\end{aligned}
$$

The Taylor series expansion for $P_{i}$ and $Q_{i}$ can be written as $(i=1,2 ; j \neq i)$ :

$$
\begin{aligned}
P_{i} & =P_{i}^{0}+\Delta P_{i} \\
& =P_{i}^{0}+\sum_{j=1}^{2} V_{j}^{0}\left(G_{i j} \cos \delta_{i j}^{0}+B_{i j} \sin \delta_{i j}^{0}\right) \Delta V_{i} \\
& +\sum_{j=1}^{2} V_{i}^{0}\left(G_{i j} \cos \delta_{i j}^{0}+B_{i j} \sin \delta_{i j}^{0}\right) \Delta V_{j} \\
& +\sum_{j=1}^{2} V_{i}^{0} V_{j}^{0}\left(-G_{i j} \sin \delta_{i j}^{0}+B_{i j} \cos \delta_{i j}^{0}\right) \Delta \delta_{i j} \\
Q_{i} & =Q_{i}^{0}+\Delta Q_{i} \\
& =Q_{i}^{0}+\sum_{j=1}^{2} V_{j}^{0}\left(G_{i j} \sin \delta_{i j}^{0}-B_{i j} \cos \delta_{i j}^{0}\right) \Delta V_{i} \\
& +\sum_{j=1}^{2} V_{i}^{0}\left(G_{i j} \sin \delta_{i j}^{0}-B_{i j} \cos \delta_{i j}^{0}\right) \Delta V_{j} \\
& +\sum_{j=1}^{2} V_{i}^{0} V_{j}^{0}\left(G_{i j} \cos \delta_{i j}^{0}+B_{i j} \sin \delta_{i j}^{0}\right) \Delta \delta_{i j}
\end{aligned}
$$

where, $\Delta \delta_{i j}=\Delta \delta_{i}-\Delta \delta_{j}$ and $\Delta \delta_{i}=\delta_{i}-\delta_{i}^{0}$. Let define $\Delta P_{m_{i}}=P_{m_{i}}+P_{f t_{i}}^{0}-P_{i}^{0}$ and $\Delta Q_{m_{i}}=Q_{m_{i}}-Q_{i}^{0}$. The system described by (12) and (13) can now be linearly represented as:

$$
\begin{aligned}
\Delta \dot{\delta}_{1} & =-k_{p_{1}} \Delta P_{m_{1}} \\
\Delta \dot{\delta}_{2} & =-k_{p_{2}} \Delta P_{m_{2}} \\
\Delta \dot{P}_{m_{1}} & =-w_{\mathrm{f}}\left(\Delta P_{m_{1}}-\Delta P_{1}\right) \\
& =-w_{\mathrm{f}} \Delta P_{m_{1}}+w_{\mathrm{f}} \Delta P_{1 \delta_{1}}^{0} \Delta \delta_{1}+w_{\mathrm{f}} \Delta P_{1 \delta_{2}}^{0} \Delta \delta_{2} \\
& +w_{\mathrm{f}} \Delta P_{1 V_{1}}^{0} \Delta V_{1}+w_{\mathrm{f}} \Delta P_{1 V_{2}}^{0} \Delta V_{2} \\
\Delta \dot{P}_{m_{2}} & =-w_{\mathrm{f}}\left(\Delta P_{m_{2}}-\Delta P_{2}\right) \\
& =-w_{\mathrm{f}} \Delta P_{m_{2}}+w_{\mathrm{f}} \Delta P_{2 \delta_{1}}^{0} \Delta \delta_{1}+w_{\mathrm{f}} \Delta P_{2 \delta_{2}}^{0} \Delta \delta_{2} \\
& +w_{\mathrm{f}} \Delta P_{2 V_{1}}^{0} \Delta V_{1}+w_{\mathrm{f}} \Delta P_{2 V_{2}}^{0} \Delta V_{2} \\
\Delta \dot{Q}_{m_{1}} & =-w_{\mathrm{f}}\left(\Delta Q_{m_{1}}-\Delta Q_{1}\right) \\
& =-w_{\mathrm{f}} \Delta Q_{m_{1}}+w_{\mathrm{f}} \Delta Q_{1 \delta_{1}}^{0} \Delta \delta_{1}+w_{\mathrm{f}} \Delta Q_{1 \delta_{2}}^{0} \Delta \delta_{2} \\
& +w_{\mathrm{f}} \Delta Q_{1 V_{1}}^{0} \Delta V_{1}+w_{\mathrm{f}} \Delta Q_{1 V_{2}}^{0} \Delta V_{2} \\
\Delta \dot{Q}_{m_{2}} & =-w_{\mathrm{f}}\left(\Delta Q_{m_{2}}-\Delta Q_{2}\right) \\
& =-w_{\mathrm{f}} \Delta Q_{m_{2}}+w_{\mathrm{f}} \Delta Q_{2 \delta_{1}}^{0} \Delta \delta_{1}+w_{\mathrm{f}} \Delta Q_{2 \delta_{2}}^{0} \Delta \delta_{2} \\
& +w_{\mathrm{f}} \Delta Q_{2 V_{1}}^{0} \Delta V_{1}+w_{\mathrm{f}} \Delta Q_{2 V_{2}}^{0} \Delta V_{2}
\end{aligned}
$$

where, the constants $\Delta P_{i \delta_{j}}^{0}, \Delta P_{i V_{j}}^{0}, \Delta Q_{i \delta_{j}}^{0}$ and $\Delta Q_{i V_{j}}^{0}$ can be obtained from (19) and (20). The eigenvalues of the 
linearized system at the equilibrium point after the change in renewable generation and load impedance are : $-12,-12$, $-12,0,-6+\mathrm{j} 15.3921$ and $-6-\mathrm{j} 15.3921$. It has been found that the system behavior, shown in Figs. 4-9, is captured by eigenvalues.

\section{Design steps of the Proposed Controller}

The controller proposed in this paper is designed in two steps:

(i) Droop gains $\left(k_{p_{1}}, k_{p_{2}}, k_{q_{1}}\right.$ and $\left.k_{q_{2}}\right)$ are selected according to the eigenvalue analysis of inductive microgrid structure such that the system is damped;

(ii) Dc gains $\left(k_{d c_{1}}\right.$ and $\left.k_{d c_{2}}\right)$ are also chosen based on the same criteria of eigenvalues of the closed-loop system mentioned in the previous step but its value should be such that proportional power sharing is possible.

\section{Controller Performance Evaluation}

The performance of the designed controller is evaluated in this section by means of stability analysis and load sharing performance so that its feasibility during both dc bus voltage and load impedance variations can be highlighted. Two VSCconnected generators and one load are considered in the test system as shown in Fig. 2.

\section{A. Sudden Change in Renewable Generation}

Let assume a situation where a sudden change occurs in the generated power of the dc micro sources at 0 second. $P_{d c_{i}}$ and $V_{d c_{i}}$ change from their set values. Now, the proposed feedback control strategy changes the output powers of microgrid in accordance with the change of $V_{d c_{i}}$ with a view to obtaining a natural balancing.

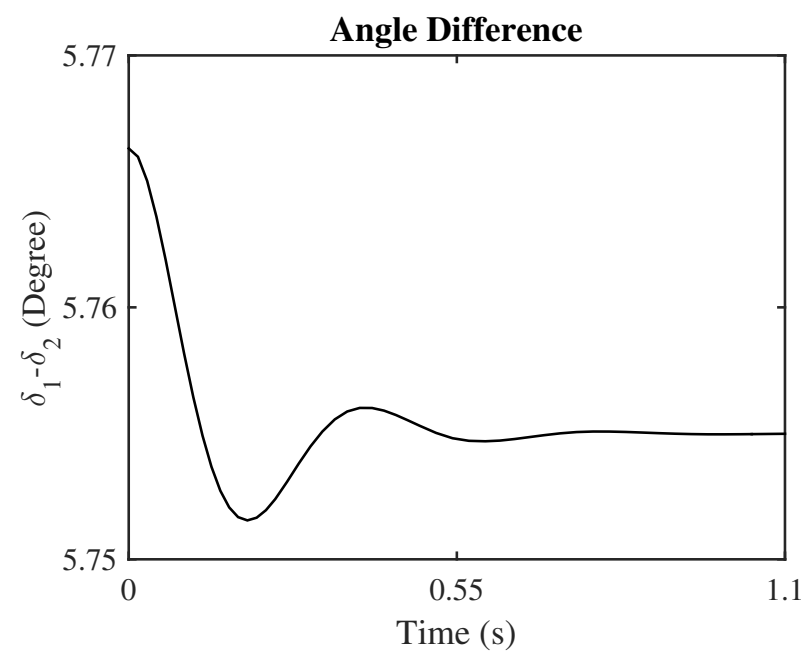

Fig. 4: Angle Difference during Variable Renewable Generation

When there is a sudden reduction, say 10 percent, in dc generation at 0 second transients are found both in the microgrid power and angle difference. The proposed control returns the system to a steady-state within 1.1 secondsthat can be noticed from Fig. 4 and Fig. 5, indicating stable operation after transients. The dc gains, $k_{d c_{i}}$, are selected for a given operating condition. In this case they are 0.0001 .

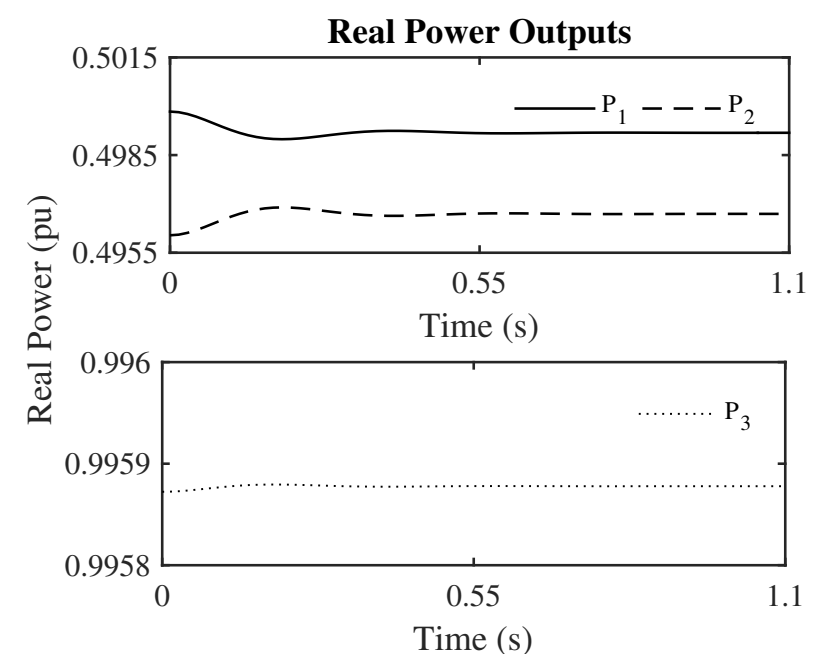

Fig. 5: Real Powers during Variable Renewable Generation

\section{B. Change in Renewable Generation and Load Impedance}

In this sub-section, the proposed control methodology is applied when load impedance change takes place, i.e., $Z_{L}$ changes from $1 \mathrm{pu}$ to $0.5+\mathrm{j} 0.25 \mathrm{pu}$, along with 10 percent increase in renewable generation.

The voltage magnitudes and angles, real and reactive powers with the proposed droop controller are shown in Fig. 6, Fig. 7, Fig. 8 and Fig. 9 respectively. It is seen from Fig. 6 that the difference between the angles of the considered microgrid approaches a constant value of $11.7945^{\circ}$, thus ensuring a stable operation.

The system is almost damped with slight initial oscillations because of real and reactive measurement sensor dynamics.

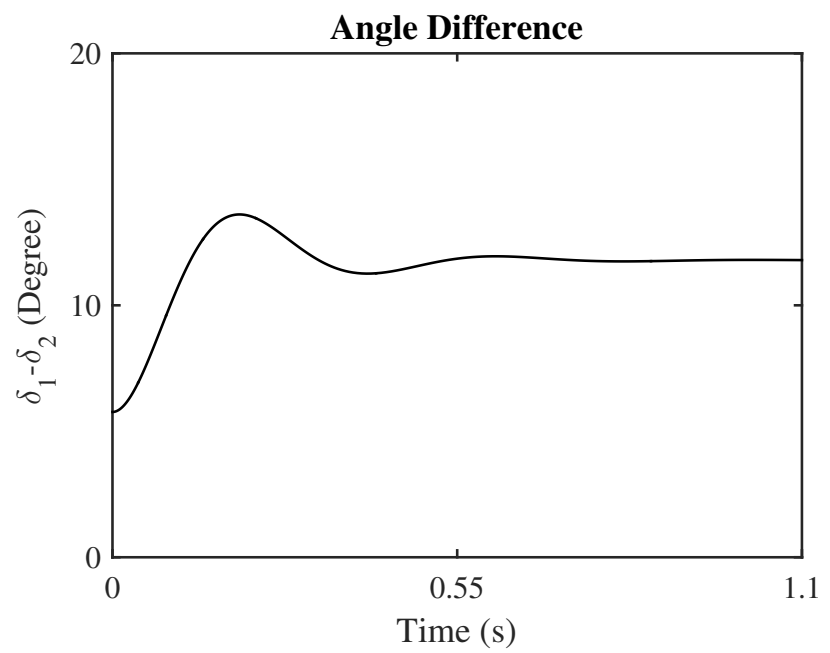

Fig. 6: Angle Difference during Variable Renewable Generation and Load Impedance 


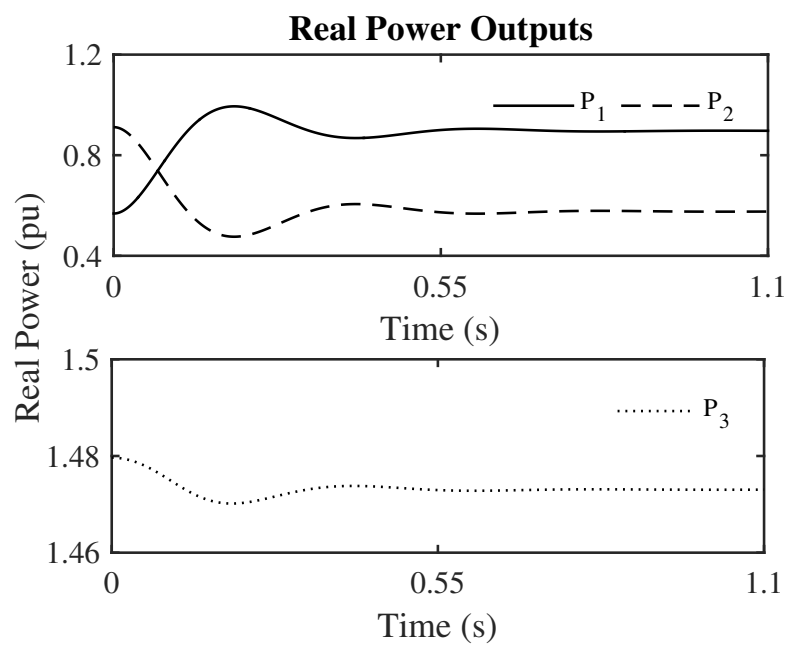

Fig. 7: Real Powers during Variable Renewable Generation and Load Impedance

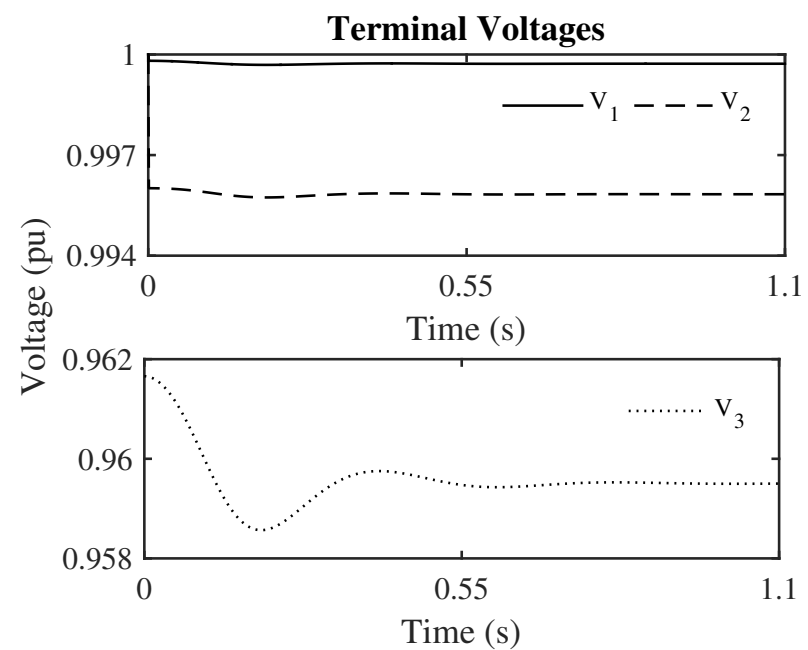

Fig. 8: Voltages during Variable Renewable Generation and Load Impedance

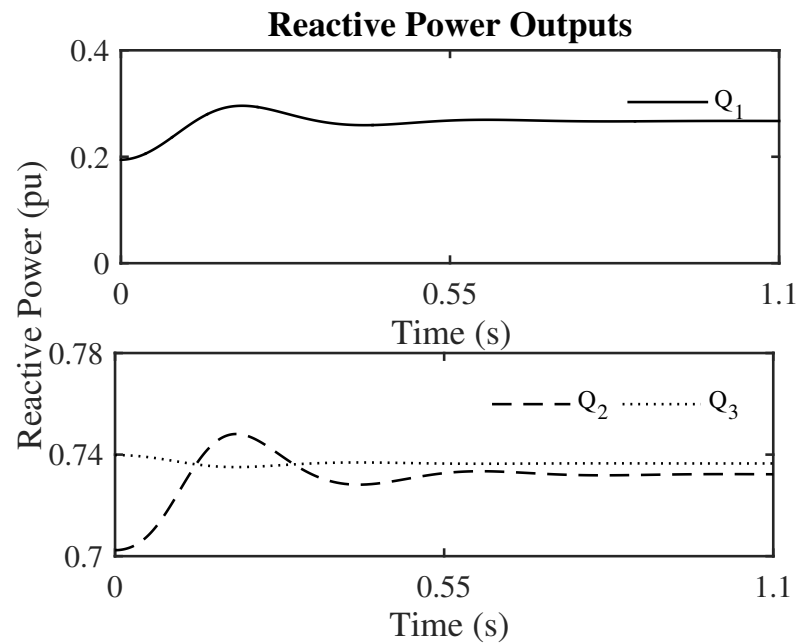

Fig. 9: Reactive Powers during Variable Renewable Generation and Load Impedance

\section{Real Power Sharing}

It is necessary that the real and reactive power are such that $\dot{\delta_{1}}=\dot{\delta_{2}}$ for stable operation of the system.

Define $\Delta P_{i}=P_{m_{i}}+P_{f t_{i}}^{0}-P_{i}^{0}$, where $i=1,2$; and from the first part of (12),

$$
\Delta P_{1}=\frac{k_{p_{2}}}{k_{p_{1}}} \Delta P_{2}=5 \Delta P_{2} \quad\left[k_{p_{1}}=1.2, k_{p_{2}}=6\right]
$$

The steady-state values of real power of both the inverterconnected voltage-sources are $P_{1}=0.8971 \mathrm{pu}$ and $P_{2}=0.5759$ pu. Now, $\Delta P_{1}=P_{1}-P_{1}^{0}=0.3971 \mathrm{pu}$ and $\Delta P_{2}=P_{2}-P_{2}^{0}=0.079 \mathrm{pu}$, giving $\Delta P_{1} \cong 5 \Delta P_{2}$, which indicates that the control analysis resembles the stability criteria and load is shared at a ratio of 5:1.

\section{Reactive Power Sharing}

The linear model of the system is further analyzed with the second part of (12) in order to share reactive power. In this case $Y_{\mathrm{b} u s}$ matrix is not reduced and $Y_{i j}=G_{i j}+j B_{i j}$. The reactive power flow of three-bus system $(i=1,2,3)$ :

$$
Q_{i}=\sum_{j=1}^{3} V_{i} V_{j}\left(G_{i j} \sin \delta_{i j}-B_{i j} \cos \delta_{i j}\right)
$$

For negligible $G_{i j}$, it can be written [16],

$$
\begin{aligned}
& Q_{1}=-V_{1} V_{3} B_{13} \cos \delta_{13}-V_{1}^{2} B_{11} \\
& Q_{2}=-V_{2} V_{3} B_{23} \cos \delta_{23}-V_{2}^{2} B_{22} \\
& Q_{3}=-V_{3} V_{1} B_{31} \cos \delta_{31}-V_{3} V_{2} B_{32} \cos \delta_{32}-V_{3}^{2} B_{33}
\end{aligned}
$$

Let there be a change in the reactive power at node- 3 of Fig. 1 and using (10), it can be written as:

$$
\begin{aligned}
\Delta Q_{1} & =k_{q_{1}} \Delta Q_{1} V_{3}^{0} B_{13} \cos \delta_{13}^{0}-V_{1}^{0}\left|\Delta V_{3}\right| B_{13} \cos \delta_{13}^{0} \\
& +V_{1}^{0} V_{3}^{0} B_{13} \sin \delta_{13}^{0}+2 k_{q_{1}} \Delta Q_{1} V_{1}^{0} B_{11} \\
\Delta Q_{2} & =k_{q_{2}} \Delta Q_{2} V_{3}^{0} B_{23} \cos \delta_{23}^{0}-V_{2}^{0}\left|\Delta V_{3}\right| B_{23} \cos \delta_{23}^{0} \\
& +V_{2}^{0} V_{3}^{0} B_{23} \sin \delta_{23}^{0}+2 k_{q_{2}} \Delta Q_{2} V_{2}^{0} B_{22} \\
\Delta Q_{3} & =k_{q_{1}} \Delta Q_{1} V_{3}^{0} B_{31} \cos \delta_{31}^{0}-V_{1}^{0}\left|\Delta V_{3}\right| B_{31} \cos \delta_{31}^{0} \\
& +V_{1}^{0} V_{3}^{0} B_{31} \sin \delta_{31}^{0}+k_{q_{2}} \Delta Q_{2} V_{3}^{0} B_{32} \cos \delta_{32}^{0} \\
& -V_{2}^{0}\left|\Delta V_{3}\right| B_{32} \cos \delta_{32}^{0}+V_{2}^{0} V_{3}^{0} B_{32} \sin \delta_{32}^{0} \\
& -2\left|\Delta V_{3}\right| V_{3}^{0} B_{33}
\end{aligned}
$$

where, $\left|\Delta V_{3}\right|=V_{3}^{0} \sqrt{\left(\cos \delta_{3}\right)^{2}+\left(-\sin \delta_{3}\right)^{2}}=V_{3}^{0}$

The simplified forms of (31) and (32) are,

$$
\begin{aligned}
\Delta Q_{1} & =\frac{-V_{1}^{0}\left|\Delta V_{3}\right| B_{13} \cos \delta_{13}^{0}+V_{1}^{0} V_{3}^{0} B_{13} \sin \delta_{13}^{0}}{1-k_{q_{1}} V_{3}^{0} B_{13} \cos \delta_{13}^{0}-2 k_{q_{1}} V_{1}^{0} B_{11}} \\
\Delta Q_{2} & =\frac{-V_{2}^{0}\left|\Delta V_{3}\right| B_{23} \cos \delta_{23}^{0}+V_{2}^{0} V_{3}^{0} B_{23} \sin \delta_{23}^{0}}{1-k_{q_{2}} V_{3}^{0} B_{13} \cos \delta_{23}^{0}-2 k_{q_{2}} V_{2}^{0} B_{22}}
\end{aligned}
$$

It is clearly found from (35) and (36) that the ratio of change in $\Delta Q_{1}$ and $\Delta Q_{2}$, due to change in $\Delta Q_{3}$, using the proposed droop strategy gives the reactive power sharing ratio. 


\section{CONCLUSION}

In this paper a decentralized droop control, with the addition of a compensator, has been proposed to enhance the feasibility of the conventional droop control scheme. A systematic procedure of implementing the proposed controller for a variable dc generation and load-based VSC-connected islanded inductive microgrid has been outlined. The designed controller guarantees system stability in both cases. Simulated results suggest that this control approach is effective enough to share active power based on droop parameters that are required for active power sharing. This paper also includes an analysis based on the linear model that makes it easier to understand reactive power sharing in microgrids depending on the reactive power droops and other microgrid system parameters. Further improvements can be made by extending the proposed controller design to include filtering approach in RES-based complex microgrids, consisting of multiple DG units and loads.

\section{REFERENCES}

[1] A. F. Zobaa, and C. Cecati, "A comprehensive review on distributed power generation," in Proc. Int. Symp. on Power Electron., Electr. Drives, Automat. and Motion, Jun. 11-13, 2008.

[2] T. L. Vandoorn, B. Meersman, J. De Kooning, and L. Vandevelde, "Transition from islanded to grid-connected mode of microgrids with voltage-based droop control," IEEE Trans. on Power Syst., vol. 28, no. 3, pp. 2545-2553, Mar. 2013.

[3] M. J. Hossain, H. R. Pota, M. A. Mahmud, and M. Aldeen, "Robust control for power sharing in microgrids with low-inertia wind and PV generators," IEEE Trans. on Sustain. Energy, vol. 6, no. 3, pp. 10671077, May 2014.

[4] T. L. Vandoorn, J. De Kooning, B. Meersman, and L. Vandevelde, "Voltage-based droop control of renewables to avoid on-off oscillations caused by overvoltages," IEEE Trans. on Power Del., vol. 28, no. 2, pp. 845-854, Feb. 2013

[5] T. L. Vandoorn, B. Meersman, J. De Kooning, and L. Vandevelde, "Analogy between conventional grid control and Islanded Microgrid control based on a global dc-link voltage droop," IEEE Trans. on Power Del., vol. 27, no. 3, pp. 1405-1414, May 2012.

[6] H. Farhangi, "The path of the smart grid," IEEE Power and Energy Mag., vol. 8, no. 1, pp. 18-28, Jan./Feb. 2010.

[7] F. Luo, Y. M. Lai, Chi K. Tse, and K. H. Loo, "A triple-droop control scheme for inverter-based microgrids," in Proc. Annual Conf. on IEEE Ind. Electron. Soc., pp. 3368-3375, Oct. 25-28, 2012.

[8] A. G. Tsikalakis, and N. D. Hatziargyriou, "Centralized control for optimizing microgrids operation," in Proc. IEEE PES Gen. Meet., pp. 1-8, Jul. 24-29, 2011.

[9] T. L. Vandoorn, B. Renders, L. Degroote, M. Meersman, and L. Vandevelde, "Power balancing in islanded microgrids by using a dcbus voltage reference," in Proc. Int. Symp. on Power Electron., Electr. Drives, Automat. and Motion, pp. 884-889, Jun. 14-16, 2010.

[10] M. A. Barik, H. R. Pota, and J. Ravishankar, "An automatic load sharing approach for a DFIG based wind generation in a microgrid," in Proc. IEEE Conf. on Ind. Electron. and Appl., pp. 589 - 594, Jun. 19-21, 2013.

[11] T. C. Green, and M. Prodanovic, "Control of inverter-based microgrids," Electric Power Syst. Research, vol. 77, no. 9, pp. 1204-1213, Jul. 2007.

[12] M. Prodanovic, and T. C. Green, "High-quality power generation through distributed control of a power park microgrid," IEEE Trans. on Ind. Electron., vol. 53, no. 5, pp. 1107-1115, Oct. 2006.

[13] J. A. P. Lopes, C. L. Moreira, and A. G. Madureira, "Defining controlstrategies for microgrids in islanded operation," IEEE Trans. on Power Syst., vol. 21, no. 2, pp. 916-924, May 2006.

[14] Y. Tan, K. M. Muttaqi, and L. G. Meegahapola, "A droop control based load sharing approach for management of renewable and nonrenewable energy sources in a remote power system," in Proc. Australian Universities Power Eng. Conf., pp. 1-6, Sep. 29-Oct. 3, 2013.
[15] T. L. Vandoorn, J. D. M. De Kooning, B. Meersman, J. M. Gurrero, and L. Vandevelde, "Automatic power-sharing modification of P/V droop controllers in low-voltage resistive microgrids," IEEE Trans. on Power Del., vol. 27, no. 4, pp. 2318-2325, Oct. 2012.

[16] H. R. Pota, "Droop control for islanded microgrids," in Proc. IEEE PES Gen. Meet., pp. 1-4, Jul. 21-25, 2013.

[17] F. Luo, Y. M. Lai, K. H. Loo, Chi K. Tse, and X. Ruan, "A generalized droop-control scheme for decentralized control of inverter-interfaced microgrids," in Proc. IEEE Symposium on Inverter-Interfaced Microgrids, pp. 1320-1323, May 19-23, 2013.

[18] B. Johnson, A. Davoudi, P. Chapman, and P. Sauer, "A unified dynamic characterization framework for microgrid systems," Electric power Components and Syst., vol. 40, no. 1, pp. 93-111, Nov. 2011.

[19] H. R. Pota, M. J. Hossain, M. A. Mahmud, R. Gadh, and R. C. Bansal, "Islanded Operation of microgrids with inverter connected renewable energy sources," in Proc. IFAC World Congress, Aug. 24-29, 2014.

[20] K. De Brabandere, B. Bolsens, J. Van den Keybus, A. Woyte, J. Driesen, and R. Belmans, "A voltage and frequency droop control method for parallel inverters," IEEE Trans. on Power Electron., vol. 22, no. 4, pp. 1107-1115, Jul. 2007.

[21] H. R. Pota, M. J. Hossain, M. A. Mahmud, and R. Gadh, "Control for microgrids with inverter connected renewable energy resources," in Proc. IEEE PES Gen. Meet., pp. 1-5, Jul. 27-31, 2014. 